\title{
A Half-Discrete Hilbert-Type Inequality in the Whole Plane with Multiparameters
}

\author{
Qunwei Ma, ${ }^{1}$ Bicheng Yang, ${ }^{2}$ and Leping $\mathrm{He}^{1}$ \\ ${ }^{1}$ College of Mathematics and Statistics, Jishou University, Hunan, Jishou 416000, China \\ ${ }^{2}$ Department of Mathematics, Guangdong University of Education, Guangzhou, Guangdong 51003, China \\ Correspondence should be addressed to Leping He; jdheleping@163.com
}

Received 2 June 2016; Accepted 1 August 2016

Academic Editor: Alberto Fiorenza

Copyright (c) 2016 Qunwei Ma et al. This is an open access article distributed under the Creative Commons Attribution License, which permits unrestricted use, distribution, and reproduction in any medium, provided the original work is properly cited.

By the use of weight functions and technique of real analysis, a new half-discrete Hilbert-type inequality in the whole plane with multiparameters and the best possible constant factor is given. Furthermore, the equivalent forms, two kinds of particular inequalities, and the operator expressions with the norm are considered.

\section{Introduction}

If $p>1,1 / p+1 / q=1, a_{m}, b_{n}>0,0<\sum_{m=1}^{\infty} a_{m}^{p}<\infty, 0<$ $\sum_{n=1}^{\infty} b_{n}^{q}<\infty$, then we have the following discrete HardyHilbert inequality (cf. [1]):

$$
\sum_{n=1}^{\infty} \sum_{m=1}^{\infty} \frac{a_{m} b_{n}}{m+n}<\frac{\pi}{\sin (\pi / p)}\left(\sum_{m=1}^{\infty} a_{m}^{p}\right)^{1 / p}\left(\sum_{n=1}^{\infty} b_{n}^{q}\right)^{1 / q}
$$

where the constant factor $\pi / \sin (\pi / p)$ is the best possible one. Assuming that $f(x), g(y) \geq 0$, satisfying $0<\int_{0}^{\infty} f^{p}(x) d x<$ $\infty$ and $0<\int_{0}^{\infty} g^{q}(y) d y<\infty$, we have the following HardyHilbert integral inequality (cf. [2]):

$$
\begin{aligned}
& \iint_{0}^{\infty} \frac{f(x) g(y)}{x+y} d x d y \\
& <\frac{\pi}{\sin (\pi / p)}\left(\int_{0}^{\infty} f^{p}(x) d x\right)^{1 / p}\left(\int_{0}^{\infty} g^{q}(y) d y\right)^{1 / q}
\end{aligned}
$$

with the best possible constant factor $\pi / \sin (\pi / p)$. Recently, half-discrete Hardy-Hilbert's inequality with the same best possible constant factor was given as follows [3]:

$$
\begin{aligned}
& \sum_{n=1}^{\infty} \int_{0}^{\infty} \frac{b_{n} f(x)}{x+n} d x \\
& \quad<\frac{\pi}{\sin (\pi / p)}\left(\int_{0}^{\infty} f^{p}(x) d x\right)^{1 / p}\left(\sum_{n=1}^{\infty} b_{n}^{q}\right)^{1 / q} .
\end{aligned}
$$

Inequalities (1), (2), and (3) are important in analysis and its applications (cf. $[2,4,5])$.

Noticing that inequalities (1) and (2) are with homogenous kernels of degree -1 , in 2009 , a survey of the study of Hilbert-type inequalities with the homogeneous kernels of degree negative numbers and some parameters is given in [6]. Recently, some inequalities with the homogenous kernels of degree 0 and nonhomogenous kernels have been studied in $[7,8]$. The other kinds of Hilbert-type inequalities are provided in [9-19]. All of the above inequalities are built in the quarter plane of the first quadrant.

In 2007, Yang [20] gave a new Hilbert-type integral inequality in the whole plane as follows:

$$
\begin{aligned}
& \iint_{-\infty}^{\infty} \frac{f(x) g(y)}{\left(1+e^{x+y}\right)^{\lambda}} d x d y<B\left(\frac{\lambda}{2}, \frac{\lambda}{2}\right) \\
& \cdot\left(\int_{-\infty}^{\infty} e^{-\lambda x} f^{2}(x) d x \int_{-\infty}^{\infty} e^{-\lambda y} g^{2}(y) d y\right)^{1 / 2},
\end{aligned}
$$


where the constant factor $B(\lambda / 2, \lambda / 2)(\lambda>0)$ is the best possible one $(B(u, v)$ is the beta function). And Zeng et al. [21, 22] also published some new Hilbert-type integral inequalities in the whole plane.

In this paper, by the use of weight functions and technique of real analysis, a new half-discrete Hilbert-type inequality in the whole plane with the best possible constant factor is built as follows: for $\rho>0,0<\sigma<\gamma(\sigma \leq 1)$,

$$
\begin{aligned}
& \sum_{|n|=1}^{\infty} \int_{-\infty}^{\infty} \frac{f(x) b_{n}}{1+\rho|n x|^{\gamma}} d x \\
& <\frac{2 \pi}{\gamma \rho^{\sigma / \gamma} \sin \pi(\sigma / \gamma)}\left[\int_{-\infty}^{\infty}|x|^{p(1-\sigma)-1} f^{p}(x) d x\right]^{1 / p} \\
& \quad \cdot\left[\sum_{|n|=1}^{\infty}|n|^{q(1-\sigma)-1} b_{n}^{q}\right]^{1 / q} .
\end{aligned}
$$

Furthermore, an extension of (5) with multiparameters is given. The equivalent forms, two kinds of particular inequalities, and the operator expressions with the norm are considered.

\section{Some Lemmas}

In the following, we agree that $\delta \in\{-1,1\}, \alpha, \beta \in(0, \pi)$, $\rho, \gamma>0$ :

$$
\begin{array}{r}
k(x, y):=\frac{1}{1+\rho\left[(|y|+y \cos \beta) /(|x|+x \cos \alpha)^{\delta}\right]^{\gamma}} \\
\quad(x, y \in \mathbf{R} \backslash\{0\}),
\end{array}
$$

wherefrom

$$
\begin{aligned}
k(x, y) & =\frac{1}{1+\rho\left[y(1+\cos \beta) /(|x|+x \cos \alpha)^{\delta}\right]^{\gamma}} \\
k(x, y) & =\frac{1}{1+\rho\left\{(|y|+y \cos \beta) /[x(1+\cos \alpha)]^{\delta}\right\}^{\gamma}}, \\
k(-x, y) & =\frac{(x>0),}{1+\rho\left\{(|y|+y \cos \beta) /[x(1-\cos \alpha)]^{\delta}\right\}^{\gamma}} \\
k(x,-y) & =\frac{1}{1+\rho\left[y(1-\cos \beta) /(|x|+x \cos \alpha)^{\delta}\right]^{\gamma}}
\end{aligned}
$$$$
(y>0) \text {. }
$$

Lemma 1 (cf. [23]). Suppose that $g(t)(>0)$ is decreasing in $\mathbf{R}_{+}$and strictly decreasing in $\left[n_{0}, \infty\right)\left(n_{0} \in \mathbf{N}\right)$, satisfying $\int_{0}^{\infty} g(t) d t \in \mathbf{R}_{+}$. One has

$$
\int_{1}^{\infty} g(t) d t<\sum_{n=1}^{\infty} g(n)<\int_{0}^{\infty} g(t) d t
$$

Lemma 2. One defines two weight functions $\omega(\sigma, n)$ and $\varpi(\sigma, x)$ as follows:

$$
\begin{aligned}
& \omega(\sigma, n):=\int_{-\infty}^{\infty} k(x, n) \frac{(|n|+n \cos \beta)^{\sigma} d x}{(|x|+x \cos \alpha)^{1+\delta \sigma}} \quad(|n| \in \mathbf{N}), \\
& \omega(\sigma, x):=\sum_{|n|=1}^{\infty} k(x, n) \frac{(|x|+x \cos \alpha)^{-\delta \sigma}}{(|n|+n \cos \beta)^{1-\sigma}}
\end{aligned}
$$

$(x \in \mathbf{R} \backslash\{0\})$,

where $\sum_{|n|=1}^{\infty} \cdots=\sum_{n=-1}^{-\infty} \cdots+\sum_{n=1}^{\infty} \cdots$. Then, for $0<\sigma<\gamma$, one has

$$
\omega(\sigma, n)=k_{\alpha}(\sigma):=\frac{2 \pi \csc ^{2} \alpha}{\gamma \rho^{\sigma / \gamma} \sin \pi(\sigma / \gamma)} \in \mathbf{R}_{+}
$$

$(|n| \in \mathbf{N})$

for $0<\sigma<\gamma(\sigma \leq 1)$, one has

$$
\begin{array}{r}
k_{\beta}(\sigma)(1-\theta(\sigma, x))<\Phi(\sigma, x)<k_{\beta}(\sigma) \\
(x \in \mathbf{R} \backslash\{0\}),
\end{array}
$$

where

$$
\theta(\sigma, x)
$$

$$
\begin{aligned}
& :=\frac{\sin \pi(\sigma / \gamma)}{\pi} \int_{0}^{\rho\left[(1+\cos \beta) /(|x|+x \cos \alpha)^{\delta}\right]^{\gamma}} \frac{u^{\sigma / \gamma-1}}{1+u} d u \\
& =O\left(\frac{1}{(|x|+x \cos \alpha)^{\delta \sigma}}\right) \in(0,1) .
\end{aligned}
$$

Proof. (i) We have

$$
\begin{aligned}
\omega(\sigma, n)= & \int_{-\infty}^{0} k(x, n) \frac{(|n|+n \cos \beta)^{\sigma}}{[x(\cos \alpha-1)]^{1+\delta \sigma}} d x \\
& +\int_{0}^{\infty} k(x, n) \frac{(|n|+n \cos \beta)^{\sigma}}{[x(\cos \alpha+1)]^{1+\delta \sigma}} d x \\
= & \int_{0}^{\infty} k(-x, n) \frac{(|n|+n \cos \beta)^{\sigma}}{[x(1-\cos \alpha)]^{1+\delta \sigma}} d x \\
& +\int_{0}^{\infty} k(x, n) \frac{(|n|+n \cos \beta)^{\sigma}}{[x(1+\cos \alpha)]^{1+\delta \sigma}} d x .
\end{aligned}
$$


Setting $u=\rho\left\{(|n|+n \cos \beta) /[x(1-\cos \alpha)]^{\delta}\right\}^{\gamma}(u=\rho\{(|n|+$ $\left.\left.n \cos \beta) /[x(1+\cos \alpha)]^{\delta}\right\}^{\gamma}\right)$ in the above first (second) integral, by simplifications, we find

$$
\begin{aligned}
\omega(\sigma, n)= & \frac{1}{(1-\cos \alpha) \gamma \rho^{\sigma / \gamma}} \int_{0}^{\infty} \frac{1}{1+u} u^{\sigma / \gamma-1} d u \\
& +\frac{1}{(1+\cos \alpha) \gamma \rho^{\sigma / \gamma}} \int_{0}^{\infty} \frac{1}{1+u} u^{\sigma / \gamma-1} d u \\
= & \frac{2 \pi \csc ^{2} \alpha}{\gamma \rho^{\sigma / \gamma} \sin \pi(\sigma / \gamma)}
\end{aligned}
$$

Hence, we have (11).

(ii) We have

$$
\begin{aligned}
\omega(\sigma, x)= & \sum_{n=-1}^{-\infty} k(x, n) \frac{(|x|+x \cos \alpha)^{-\delta \sigma}}{(|n|+n \cos \beta)^{1-\sigma}} \\
& +\sum_{n=1}^{\infty} k(x, n) \frac{(|x|+x \cos \alpha)^{-\delta \sigma}}{(|n|+n \cos \beta)^{1-\sigma}} \\
= & \frac{(|x|+x \cos \alpha)^{-\delta \sigma}}{(1-\cos \beta)^{1-\sigma}} \sum_{n=1}^{\infty} \frac{k(x,-n)}{n^{1-\sigma}} \\
& +\frac{(|x|+x \cos \alpha)^{-\delta \sigma}}{(1+\cos \beta)^{1-\sigma}} \sum_{n=1}^{\infty} \frac{k(x, n)}{n^{1-\sigma}} .
\end{aligned}
$$

Since, for $0<\sigma<\gamma(\sigma \leq 1)$, both $k(x,-y) / y^{1-\sigma}$ and $k(x, y) / y^{1-\sigma}$ are strictly decreasing in $(0, \infty)$, satisfying

$$
\begin{gathered}
\frac{d}{d y} \frac{k(x,-y)}{y^{1-\sigma}}<0, \\
\frac{d}{d y} \frac{k(x, y)}{y^{1-\sigma}}<0,
\end{gathered}
$$

by (16) and (8), we obtain

$$
\begin{aligned}
\omega(\sigma, x)< & \frac{(|x|+x \cos \alpha)^{-\delta \sigma}}{(1-\cos \beta)^{1-\sigma}} \int_{0}^{\infty} \frac{k(x,-y)}{y^{1-\sigma}} d y \\
& +\frac{(|x|+x \cos \alpha)^{-\delta \sigma}}{(1+\cos \beta)^{1-\sigma}} \int_{0}^{\infty} \frac{k(x, y)}{y^{1-\sigma}} d y .
\end{aligned}
$$

Setting $u=\rho\left[y(1-\cos \beta) /(|x|+x \cos \alpha)^{\delta}\right]^{\gamma}(u=\rho[y(1+$ $\left.\left.\cos \beta) /(|x|+x \cos \alpha)^{\delta}\right]^{\gamma}\right)$ in the above first (second) integral, by simplifications, we find

$$
\varpi(\sigma, x)<\frac{2 \pi \csc ^{2} \beta}{\gamma \rho^{\sigma / \gamma} \sin \pi(\sigma / \gamma)}=k_{\beta}(\sigma) .
$$

By (16) and (8), we still have

$$
\begin{aligned}
\varpi(\sigma, x)> & \frac{(|x|+x \cos \alpha)^{-\delta \sigma}}{(1-\cos \beta)^{1-\sigma}} \int_{1}^{\infty} \frac{k(x,-y)}{y^{1-\sigma}} d y \\
& +\frac{(|x|+x \cos \alpha)^{-\delta \sigma}}{(1+\cos \beta)^{1-\sigma}} \int_{1}^{\infty} \frac{k(x, y)}{y^{1-\sigma}} d y .
\end{aligned}
$$

Setting $u=\rho\left[y(1-\cos \beta) /(|x|+x \cos \alpha)^{\delta}\right]^{\gamma}(u=\rho[y(1+$ $\left.\left.\cos \beta) /(|x|+x \cos \alpha)^{\delta}\right]^{\gamma}\right)$ in the above first (second) integral, by simplifications, we obtain

$$
\begin{aligned}
& \varpi(\sigma, x)>\frac{1}{\gamma \rho^{\sigma / \gamma}(1-\cos \beta)} \\
& \cdot \int_{\rho\left[(1-\cos \beta) /(|x|+x \cos \alpha)^{\delta}\right]^{\gamma}}^{\infty} \frac{u^{\sigma / \gamma-1}}{1+u} d u \\
& +\frac{1}{\gamma \rho^{\sigma / \gamma}(1+\cos \beta)} \\
& \cdot \int_{\rho\left[(1+\cos \beta) /(|x|+x \cos \alpha)^{\delta}\right]^{\gamma}}^{\infty} \frac{u^{\sigma / \gamma-1}}{1+u} d u \geq \frac{2 \csc ^{2} \beta}{\gamma \rho^{\sigma / \gamma}} \\
& \cdot \int_{\rho\left[(1+\cos \beta) /(|x|+x \cos \alpha)^{\delta}\right]^{\gamma}}^{\infty} \frac{u^{\sigma / \gamma-1}}{1+u} d u=k_{\beta}(\sigma) \\
& \cdot(1-\theta(\sigma, x))>0,
\end{aligned}
$$

where $\theta(\sigma, x)$ is indicated by (13). We find

$$
\begin{aligned}
0 & <\theta(\sigma, x) \\
& \leq \frac{\sin \pi(\sigma / \gamma)}{\pi} \int_{0}^{\rho\left[(1+\cos \beta) /(|x|+x \cos \alpha)^{\delta}\right]^{\gamma}} u^{\sigma / \gamma-1} d u \\
& =\frac{\gamma \rho^{\sigma / \gamma} \sin \pi(\sigma / \gamma)}{\sigma \pi}\left[\frac{1+\cos \beta}{(|x|+x \cos \alpha)^{\delta}}\right]^{\sigma}
\end{aligned}
$$

and then (12) and (13) follow.

Lemma 3. For $\varepsilon>0$, setting $E_{\delta}:=\{x \in \mathbf{R} \backslash\{0\} ; 1 /(|x|+$ $\left.x \cos \alpha)^{\delta} \geq 1\right\}$, one has

$$
H_{\delta}:=\int_{E_{\delta}} \frac{1}{(|x|+x \cos \alpha)^{1+\delta \varepsilon}} d x=\frac{2}{\varepsilon} \csc ^{2} \alpha .
$$

Proof. Setting

$$
\begin{aligned}
& E_{\delta}^{+}:=\left\{x>0 ; \frac{1}{[x(1+\cos \alpha)]^{\delta}} \geq 1\right\}, \\
& E_{\delta}^{-}:=\left\{x<0 ; \frac{1}{[-x(1-\cos \alpha)]^{\delta}} \geq 1\right\},
\end{aligned}
$$


it follows that $E_{\delta}=E_{\delta}^{+} \cup E_{\delta}^{-}$. We find

$$
\begin{aligned}
H_{\delta}= & \int_{E_{\delta}^{+}} \frac{d x}{[x(1+\cos \alpha)]^{1+\delta \varepsilon}} \\
& +\int_{E_{\delta}^{-}} \frac{d x}{[-x(1-\cos \alpha)]^{1+\delta \varepsilon}} .
\end{aligned}
$$

Setting $u=[x(1+\cos \alpha)]^{\delta}\left(u=[-x(1-\cos \alpha)]^{\delta}\right)$ in the above first (second) integral, we obtain

$$
H_{\delta}=\left(\frac{1}{1+\cos \alpha}+\frac{1}{1-\cos \alpha}\right) \int_{1}^{\infty} \frac{d u}{u^{1+\varepsilon}}=\frac{2}{\varepsilon} \csc ^{2} \alpha .
$$

Hence we have (23).

Lemma 4. For $\varepsilon>0$, setting

$$
H_{\varepsilon}(\beta):=\sum_{|n|=1}^{\infty} \frac{1}{(|n|+n \cos \beta)^{1+\varepsilon}}
$$

we have

$$
H_{\varepsilon}(\beta)<\frac{1}{\varepsilon}\left(2 \csc ^{2} \beta+o(1)\right)(\varepsilon+1) \quad\left(\varepsilon \longrightarrow 0^{+}\right) .
$$

Proof. We have

$$
\begin{aligned}
H_{\varepsilon}(\beta)= & \sum_{n=-1}^{-\infty} \frac{1}{[n(\cos \beta-1)]^{1+\varepsilon}} \\
& +\sum_{n=1}^{\infty} \frac{1}{[n(\cos \beta+1)]^{1+\varepsilon}} \\
= & {\left[\frac{1}{(1-\cos \beta)^{1+\varepsilon}}+\frac{1}{(1+\cos \beta)^{1+\varepsilon}}\right] \sum_{n=1}^{\infty} \frac{1}{n^{1+\varepsilon}} . }
\end{aligned}
$$

$$
\begin{aligned}
& I:=\sum_{|n|=1}^{\infty} \int_{-\infty}^{\infty} \frac{f(x) b_{n}}{1+\rho\left((|n|+n \cos \beta) /(|x|+x \cos \alpha)^{\delta}\right)^{\gamma}} d x<k_{\alpha, \beta}(\sigma)\left[\int_{-\infty}^{\infty}(|x|+x \cos \alpha)^{p(1+\delta \sigma)-1} f^{p}(x) d x\right]^{1 / p} \\
& {\left[\sum_{|n|=1}^{\infty}(|n|+n \cos \beta)^{q(1-\sigma)-1} b_{n}^{q}\right]^{1 / q}} \\
& J_{1}:=\left\{\sum_{|n|=1}^{\infty}(|n|+n \cos \beta)^{p \sigma-1}\left[\int_{-\infty}^{\infty} \frac{f(x) d x}{1+\rho\left((|n|+n \cos \beta) /(|x|+x \cos \alpha)^{\delta}\right)^{\gamma}}\right]^{p}\right\}^{1 / p}<k_{\alpha, \beta}(\sigma)\left[\int_{-\infty}^{\infty}(|x|\right. \\
& \left.+x \cos \alpha)^{p(1+\delta \sigma)-1} f^{p}(x) d x\right]^{1 / p} \\
& J_{2}:=\left\{\int_{-\infty}^{\infty} \frac{1}{(|x|+x \cos \alpha)^{q \delta \sigma+1}}\left[\sum_{|n|=1}^{\infty} \frac{b_{n}}{1+\rho\left((|n|+n \cos \beta) /(|x|+x \cos \alpha)^{\delta}\right)^{\gamma}}\right]^{q} d x\right\}^{1 / q}<k_{\alpha, \beta}(\sigma)\left[\sum_{|n|=1}^{\infty}(|n|\right. \\
& \left.+n \cos \beta)^{q(1-\sigma)-1} b_{n}^{q}\right]^{1 / q}
\end{aligned}
$$


In particular, for $\alpha=\beta=\pi / 2$, we have the following equivalent inequalities:

$$
\begin{aligned}
& \sum_{|n|=1}^{\infty} \int_{-\infty}^{\infty} \frac{f(x) b_{n}}{1+\rho\left(|n| /|x|^{\delta}\right)^{\gamma}} d x \\
& <\frac{2 \pi}{\gamma \rho^{\sigma / \gamma} \sin (\pi \sigma / \gamma)}\left[\int_{-\infty}^{\infty}|x|^{p(1+\delta \sigma)-1}\right. \\
& \left.f^{p}(x) d x\right]^{1 / p}\left[\sum_{|n|=1}^{\infty}|n|^{q(1-\sigma)-1} b_{n}^{q}\right]^{1 / q}, \\
& \left\{\sum_{|n|=1}^{\infty}|n|^{p \sigma-1}\left[\int_{-\infty}^{\infty} \frac{f(x)}{1+\rho\left(|n| /|x|^{\delta}\right)^{\gamma}} d x\right]^{p}\right\}^{1 / p} \\
& <\frac{2 \pi}{\gamma \rho^{\sigma / \gamma} \sin (\pi \sigma / \gamma)}\left[\int_{-\infty}^{\infty}|x|^{p(1+\delta \sigma)-1}\right. \\
& \left.f^{p}(x) d x\right]^{1 / p}, \\
& \left\{\int_{-\infty}^{\infty} \frac{1}{|x|^{q \delta \sigma+1}}\left[\sum_{|n|=1}^{\infty} \frac{b_{n}}{1+\rho\left(|n| /|x|^{\delta}\right)^{\gamma}}\right]^{q} d x\right\}^{1 / q} \\
& <\frac{2 \pi}{\gamma \rho^{\sigma / \gamma} \sin (\pi \sigma / \gamma)}\left[\sum_{|n|=1}^{\infty}|n|^{q(1-\sigma)-1} b_{n}^{q}\right]^{1 / q} .
\end{aligned}
$$

Proof. By Hölder's inequality (cf. [24]) and (9), we find

$$
\begin{aligned}
&\left(\int_{-\infty}^{\infty} k(x, n) f(x) d x\right)^{p} \\
&=\left[\int_{-\infty}^{\infty} k(x, n) \frac{(|x|+x \cos \alpha)^{(1+\delta \sigma) / q}}{(|n|+n \cos \beta)^{(1-\sigma) / p}} f(x)\right. \\
& \cdot \frac{(|n|+n \cos \beta)^{(1-\sigma) / p}}{\left.(|x|+x \cos \alpha)^{(1+\delta \sigma) / q} d x\right]^{p} \leq \int_{-\infty}^{\infty} k(x, n)} \\
& . \frac{(|x|+x \cos \alpha)^{(1+\delta \sigma)(p-1)}}{(|n|+n \cos \beta)^{1-\sigma}} f^{p}(x) d x \\
& . {\left[\int_{-\infty}^{\infty} k(x, n) \frac{(|n|+n \cos \beta)^{(1-\sigma)(q-1)}}{(|x|+x \cos \alpha)^{1+\delta \sigma}} d x\right]^{p-1} } \\
&= \frac{\omega^{p-1}(\sigma, n)}{(|n|+n \cos \beta)^{p \sigma-1} \int_{-\infty}^{\infty} k(x, n)} \\
& \frac{(|x|+x \cos \alpha)^{(1+\delta \sigma)(p-1)}}{(|n|+n \cos \beta)^{1-\sigma}} f^{p}(x) d x .
\end{aligned}
$$

Then by (11) and Lebesgue term-by-term integration theorem (cf. [25]), in view of (10), we find

$$
\begin{aligned}
J_{1} \leq & k_{\alpha}^{1 / q}(\sigma)\left[\sum_{|n|=1}^{\infty} \int_{-\infty}^{\infty} k(x, n)\right. \\
& \left.\cdot \frac{(|x|+x \cos \alpha)^{(1+\delta \sigma)(p-1)}}{(|n|+n \cos \beta)^{1-\sigma}} f^{p}(x) d x\right]^{1 / p}=k_{\alpha}^{1 / q}(\sigma) \\
\cdot & {\left[\int_{-\infty}^{\infty} \sum_{|n|=1}^{\infty} k(x, n)\right.} \\
& \left.\cdot \frac{(|x|+x \cos \alpha)^{(1+\delta \sigma)(p-1)}}{(|n|+n \cos \beta)^{1-\sigma}} f^{p}(x) d x\right]^{1 / p}=k_{\alpha}^{1 / q}(\sigma) \\
& \cdot\left[\int_{-\infty}^{\infty} \omega(\sigma, x)(|x|+x \cos \alpha)^{p(1+\delta \sigma)-1} f^{p}(x) d x\right]^{1 / p} .
\end{aligned}
$$

Then, by (12), we have (34).

By Hölder's inequality (cf. [24]), we have

$$
\begin{aligned}
I= & \sum_{|n|=1}^{\infty}\left[(|n|+n \cos \beta)^{-1 / p+\sigma} \int_{-\infty}^{\infty} k(x, n) f(x) d x\right] \\
& \cdot\left[(|n|+n \cos \beta)^{1 / p-\sigma} b_{n}\right] \\
& \leq J_{1}\left[\sum_{|n|=1}^{\infty}(|n|+n \cos \beta)^{q(1-\sigma)-1} b_{n}^{q}\right]^{1 / q} .
\end{aligned}
$$

Then, by (34), we have (33). On the other hand, assuming that (33) is valid, we set

$$
b_{n}:=(|n|+n \cos \beta)^{p \sigma-1}\left[\int_{-\infty}^{\infty} k(x, n) f(x) d x\right]^{p-1}
$$

$(|n| \in \mathbf{N})$.

Then we find

$$
J_{1}=\left[\sum_{|n|=1}^{\infty}(|n|+n \cos \beta)^{q(1-\sigma)-1} b_{n}^{q}\right]^{1 / p} .
$$

In view of (40), it follows that $J_{1}<\infty$. If $J_{1}=0$, and then (34) is trivially valid; if $J_{1}>0$, then, by (33), we have

$$
\begin{gathered}
\sum_{|n|=1}^{\infty}(|n|+n \cos \beta)^{q(1-\sigma)-1} b_{n}^{q}=J_{1}^{p}=I<k_{\alpha, \beta}(\sigma) \\
\cdot\left[\int_{-\infty}^{\infty}(|x|+x \cos \alpha)^{p(1+\delta \sigma)-1} f^{p}(x) d x\right]^{1 / p} \\
\cdot\left[\sum_{|n|=1}^{\infty}(|n|+n \cos \beta)^{q(1-\sigma)-1} b_{n}^{q}\right]^{1 / q},
\end{gathered}
$$




$$
\begin{gathered}
{\left[\sum_{|n|=1}^{\infty}(|n|+n \cos \beta)^{q(1-\sigma)-1} b_{n}^{q}\right]^{1 / p}=J_{1}<k_{\alpha, \beta}(\sigma)} \\
\cdot\left[\int_{-\infty}^{\infty}(|x|+x \cos \alpha)^{p(1+\delta \sigma)-1} f^{p}(x) d x\right]^{1 / p}
\end{gathered}
$$

namely, (34) holds, which is equivalent to (33).

In the same way of obtaining (40), we have

$$
\begin{aligned}
J_{2} \leq & k_{\beta}^{1 / p}(\sigma) \\
& \cdot\left[\sum_{|n|=1}^{\infty} \omega(\sigma, n)(|n|+n \cos \beta)^{q(1-\sigma)-1} b_{n}^{q}\right]^{1 / q} .
\end{aligned}
$$

We have proved that (33) is valid. Setting

$$
\begin{array}{r}
f(x):=\int_{-\infty}^{\infty} \frac{1}{(|x|+x \cos \alpha)^{q \delta \sigma+1}}\left[\sum_{|n|=1}^{\infty} k(x, n) b_{n}\right]^{q-1} \\
(x \in \mathbf{R} \backslash\{0\}),
\end{array}
$$

it follows that

$$
J_{2}=\left[\int_{-\infty}^{\infty}(|x|+x \cos \alpha)^{p(1+\delta \sigma)-1} f^{p}(x) d x\right]^{1 / q}
$$

and, in view of (45), $J_{2}<\infty$. If $J_{2}=0$, then (35) is trivially valid; if $J_{2}>0$, then, by (33), we have

$$
\begin{gathered}
\int_{-\infty}^{\infty}(|x|+x \cos \alpha)^{p(1+\delta \sigma)-1} f^{p}(x) d x=J_{2}^{q}=I \\
<k_{\alpha, \beta}(\sigma) \\
\cdot\left[\int_{-\infty}^{\infty}(|x|+x \cos \alpha)^{p(1+\delta \sigma)-1} f^{p}(x) d x\right]^{1 / p} \\
\cdot\left[\sum_{|n|=1}^{\infty}(|n|+n \cos \beta)^{q(1-\sigma)-1} b_{n}^{q}\right]^{1 / q}, \\
{\left[\int_{-\infty}^{\infty}(|x|+x \cos \alpha)^{p(1+\delta \sigma)-1} f^{p}(x) d x\right]^{1 / q}=J_{2}} \\
\quad<k_{\alpha, \beta}(\sigma)\left[\sum_{|n|=1}^{\infty}(|n|+n \cos \beta)^{q(1-\sigma)-1} b_{n}^{q}\right]^{1 / q} ;
\end{gathered}
$$

namely, (35) follows.
On the other hand, assuming that (35) is valid, by Hölder's inequality (cf. [24]) and in the same way of obtaining (41), we have

$$
I \leq\left[\int_{-\infty}^{\infty}(|x|+x \cos \alpha)^{p(1+\delta \sigma)-1} f^{p}(x) d x\right]^{1 / p} J_{2}
$$

Then, by (35), we have (33), which is equivalent to (35).

Therefore, inequalities (33), (34), and (35) are equivalent.

Theorem 6. As regards the assumptions of Theorem 5, the constant factor $k_{\alpha, \beta}(\sigma)$ in (33), (34), and (35) is the best possible one.

Proof. For $0<\varepsilon<q \sigma$, we set $\widetilde{\sigma}=\sigma-\varepsilon / q \in(0,1)(\widetilde{\sigma}<\gamma)$,

$$
\widetilde{f}(x):= \begin{cases}\frac{1}{(|x|+x \cos \alpha)^{\delta(\sigma+\varepsilon / p)+1},} & x \in E_{\delta}, \\ 0, & x \in \mathbf{R} \backslash E_{\delta},\end{cases}
$$

and $\widetilde{b}_{n}:=(|n|+n \cos \beta)^{(\sigma-\varepsilon / q)-1},|n| \in \mathbf{N}$. Then, by (23) and (28), we find

$$
\begin{aligned}
\widetilde{I}_{1}: & =\left[\int_{-\infty}^{\infty}(|x|+x \cos \alpha)^{p(1+\delta \sigma)-1} \tilde{f}^{p}(x) d x\right]^{1 / p} \\
& \cdot\left[\sum_{|n|=1}^{\infty}(|n|+n \cos \beta)^{q(1-\sigma)-1} \widetilde{b}_{n}^{q}\right]^{1 / q} \\
& =\left[\int_{-\infty}^{\infty} \frac{d x}{(|x|+x \cos \alpha)^{\delta \varepsilon+1}}\right]^{1 / p} \\
& \cdot\left[\sum_{|n|=1}^{\infty} \frac{1}{(|n|+n \cos \beta)^{\varepsilon+1}}\right]^{1 / q} \leq \frac{1}{\varepsilon}\left(2 \csc ^{2} \alpha\right)^{1 / p} \\
& \cdot\left[\left(2 \csc ^{2} \beta+o(1)\right)(\varepsilon+1)\right]^{1 / q} .
\end{aligned}
$$

By (12) and (23), we still have

$$
\begin{aligned}
\widetilde{I}:= & \sum_{|n|=1}^{\infty} \int_{-\infty}^{\infty} k(x, n) \tilde{f}(x) \tilde{b}_{n} d x \\
& =\int_{E_{\delta}} \sum_{|n|=1}^{\infty} k(x, n) \frac{(|x|+x \cos \alpha)^{-\delta(\widetilde{\sigma}+\varepsilon)-1}}{(|n|+n \cos \beta)^{1-\widetilde{\sigma}}} d x \\
& =\int_{E_{\delta}} \frac{\omega(\widetilde{\sigma}, x) d x}{(|x|+x \cos \alpha)^{\delta \varepsilon+1}} \geq k_{\beta}(\widetilde{\sigma})
\end{aligned}
$$




$$
\begin{aligned}
& \cdot \int_{E_{\delta}} \frac{1-\theta(\widetilde{\sigma}, x)}{(|x|+x \cos \alpha)^{\delta \varepsilon+1}} d x=k_{\beta}(\widetilde{\sigma}) \\
& \cdot\left[\int_{E_{\delta}} \frac{d x}{(|x|+x \cos \alpha)^{\delta \varepsilon+1}}\right. \\
& \left.-\int_{E_{\delta}} \frac{d x}{O\left((|x|+x \cos \alpha)^{\delta(\sigma+\varepsilon / p)+1}\right)}\right]=\frac{1}{\varepsilon} k_{\beta}(\sigma \\
& \left.-\frac{\varepsilon}{q}\right)\left(2 \csc ^{2} \alpha-\varepsilon O(1)\right) .
\end{aligned}
$$

If the constant factor $k_{\alpha, \beta}(\sigma)$ in (33) is not the best possible one, then, there exists a positive number $k$ with $k_{\alpha, \beta}(\sigma)>k$, such that (33) is still valid when replacing $k_{\alpha, \beta}(\sigma)$ by $k$. Then, in particular, we have $\varepsilon \widetilde{I}<\varepsilon k \widetilde{I}_{1}$; namely,

$$
\begin{aligned}
& k_{\beta}\left(\sigma-\frac{\varepsilon}{q}\right)\left(2 \csc ^{2} \alpha-\varepsilon O(1)\right) \\
& \quad<k\left(2 \csc ^{2} \alpha\right)^{1 / p}\left[\left(2 \csc ^{2} \beta+o(1)\right)(\varepsilon+1)\right]^{1 / q}
\end{aligned}
$$

It follows that $2 k_{\beta}(\sigma) \csc ^{2} \alpha \leq 2 k \csc ^{2 / p} \alpha \csc ^{2 / q} \beta\left(\varepsilon \rightarrow 0^{+}\right)$, and then

$$
k_{\alpha, \beta}(\sigma)=\frac{2 \pi \csc ^{2 / q} \alpha \csc ^{2 / p} \beta}{\gamma \rho^{\sigma / \gamma} \sin \pi(\sigma / \gamma)} \leq k,
$$

which contradicts the fact that $k_{\alpha, \beta}(\sigma)>k$. Hence, the constant factor $k_{\alpha, \beta}(\sigma)$ in (33) is the best possible one.

The constant factor $k_{\alpha, \beta}(\sigma)$ in (34) ((35)) is still the best possible one. Otherwise, we would reach a contradiction by (41) ((49)) that the constant factor $k_{\alpha, \beta}(\sigma)$ in (33) is not the best possible one.

\section{Operator Expressions}

Suppose that $p>1$ and $1 / p+1 / q=1$. We set the following functions:

$$
\begin{aligned}
& \Phi(x):=(|x|+x \cos \alpha)^{p(1+\delta \sigma)-1}, \\
& \Psi(n):=(|n|+n \cos \beta)^{q(1-\sigma)-1},
\end{aligned}
$$

wherefrom $\Phi^{1-q}(x)=(|x|+x \cos \alpha)^{-q \delta \sigma-1}$ and

$$
\Psi^{1-p}(n)=(|n|+n \cos \beta)^{p \sigma-1}
$$

Define the following real weight normed linear spaces:

$$
\begin{aligned}
& L_{p, \Phi}(\mathbf{R}):=\left\{f ;\|f\|_{p, \Phi}:=\left(\int_{-\infty}^{\infty} \Phi(x)|f(x)|^{p} d x\right)^{1 / p}\right. \\
& <\infty\} \text {, } \\
& L_{q, \Phi^{1-q}}(\mathbf{R}):=\left\{h ;\|h\|_{q, \Phi^{1-q}}\right. \\
& \left.:=\left(\int_{-\infty}^{\infty} \Phi^{1-q}(x)|h(x)|^{q} d x\right)^{1 / q}<\infty\right\}, \\
& l_{q, \Psi}:=\left\{b=\left\{b_{n}\right\}_{|n|=1}^{\infty} ;\|b\|_{q, \Psi}:=\left(\sum_{|n|=1}^{\infty} \Psi(n)\left|b_{n}\right|^{q}\right)^{1 / q}\right. \\
& <\infty\} \\
& l_{p, \Psi^{1-p}}:=\left\{c=\left\{c_{n}\right\}_{|n|=1}^{\infty} ;\|c\|_{p, \Psi^{1-p}}\right. \\
& \left.:=\left(\sum_{|n|=1}^{\infty} \Psi^{1-p}(n)\left|c_{n}\right|^{p}\right)^{1 / p}<\infty\right\}
\end{aligned}
$$

(a) In view of Theorem 5 , for $f \in L_{p, \Phi}(\mathbf{R})$, setting

$$
H^{(1)}(n):=\int_{-\infty}^{\infty} k(x, n)|f(x)| d x \quad(|n| \in \mathbf{N}),
$$

by (34), we have

$$
\begin{aligned}
\left\|H^{(1)}\right\|_{p, \Psi^{1-p}} & =\left[\sum_{|n|=1}^{\infty} \Psi^{1-p}(n)\left(H^{(1)}(n)\right)^{p}\right]^{1 / p} \\
& <k_{\alpha, \beta}(\sigma)\|f\|_{p, \Phi}<\infty
\end{aligned}
$$

namely, $H^{(1)} \in l_{p, \Psi^{1-p}}$.

Definition 7. Define a half-discrete Hilbert-type operator in the whole plane $T^{(1)}: L_{p, \Phi}(\mathbf{R}) \rightarrow l_{p, \Psi^{1-p}}$ as follows: for any $f \in L_{p, \Phi}(\mathbf{R})$, there exists a unique representation $T^{(1)} f=$ $H^{(1)} \in l_{p, \Psi^{1-p}}$, satisfying, for any $|n| \in \mathbf{N},\left(T^{(1)} f\right)(n)=$ $H^{(1)}(n)$.

In view of (59), it follows that $\left\|T^{(1)} f\right\|_{p, \Psi^{1-p}}=\left\|H^{(1)}\right\|_{p, \Psi^{1-p}}$ $\leq k_{\alpha, \beta}\|f\|_{p, \Phi}$, and then the operator $T^{(1)}$ is bounded satisfying

$$
\left\|T^{(1)}\right\|:=\sup _{f(\neq \theta) \in L_{p, \Phi}(\mathbf{R})} \frac{\left\|T^{(1)} f\right\|_{p, \Psi^{1-p}}}{\|f\|_{p, \Phi}} \leq k_{\alpha, \beta}(\sigma) .
$$

Since the constant factor $k_{\alpha, \beta}(\sigma)$ in (59) is the best possible one, we have

$$
\left\|T^{(1)}\right\|=k_{\alpha, \beta}(\sigma)=\frac{2 \pi \csc ^{2 / q} \alpha \csc ^{2 / p} \beta}{\gamma \rho^{\sigma / \gamma} \sin \pi(\sigma / \gamma)} .
$$


If we define the formal inner product of $T^{(1)} f$ and $b(\epsilon$ $\left.l_{q, \Psi}\right)$ as follows:

$$
\left(T^{(1)} f, b\right):=\sum_{|n|=1}^{\infty}\left(\int_{-\infty}^{\infty} k(x, n) f(x) d x\right) b_{n}
$$

then we can rewrite (33) and (34) as follows:

$$
\begin{aligned}
\left(T^{(1)} f, b\right) & <\left\|T^{(1)}\right\| \cdot\|f\|_{p, \Psi}\|b\|_{q, \Phi}, \\
\left\|T^{(1)} f\right\|_{p, \Psi^{1-p}} & <\left\|T^{(1)}\right\| \cdot\|f\|_{p, \Phi} .
\end{aligned}
$$

(b) In view of Theorem 5 , for $b \in l_{q, \Psi}$, setting

$$
H^{(2)}(x):=\sum_{|n|=1}^{\infty} k(x, n) b_{n} \quad(x \in \mathbf{R} \backslash\{0\}),
$$

then, by (35), we have

$$
\begin{aligned}
\left\|H^{(2)}\right\|_{q, \Phi^{1-q}} & =\left[\int_{-\infty}^{\infty} \Phi^{1-q}(x)\left(H^{(2)}(x)\right)^{q} d x\right]^{1 / q} \\
& <k_{\alpha, \beta}(\sigma)\|b\|_{q, \Psi}<\infty ;
\end{aligned}
$$

namely, $H^{(2)} \in L_{q, \Psi^{1-q}}(\mathbf{R})$.

Definition 8. Define a half-discrete Hilbert-type operator in the whole plane $T^{(2)}: l_{q, \Psi} \rightarrow L_{q, \Psi^{1-q}}(\mathbf{R})$ as follows: for any $b \in l_{q, \Psi}$, there exists a unique representation $T^{(2)} b=H^{(2)} \in$ $L_{q, \Psi^{1-q}}(\mathbf{R})$, satisfying, for any $x \in \mathbf{R} \backslash\{0\},\left(T^{(2)} b\right)(x)=H^{(2)}(x)$.

In view of (65), it follows that $\left\|T^{(2)} b\right\|_{q, \Phi^{1-q}}=\left\|H^{(2)}\right\|_{q, \Phi^{1-q}}$ $\leq k_{\alpha, \beta}(\sigma)\|b\|_{q, \Psi}$, and then the operator $T^{(2)}$ is bounded, satisfying

$$
\left\|T^{(2)}\right\|:=\sup _{b(\neq \theta) \in l_{q, \Psi}} \frac{\left\|T^{(2)} b\right\|_{q, \Phi^{1-q}}}{\|b\|_{q, \Psi}} \leq k_{\alpha, \beta}(\sigma) .
$$

Since the constant factor $k_{\alpha, \beta}(\sigma)$ in (65) is the best possible one, we have

$$
\left\|T^{(2)}\right\|=k_{\alpha, \beta}(\sigma)=\left\|T^{(1)}\right\| .
$$

If we define the formal inner product of $T^{(2)} b$ and $f(\epsilon$ $\left.L_{p, \Phi}(\mathbf{R})\right)$ as follows:

$$
\left(T^{(2)} b, f\right):=\int_{-\infty}^{\infty}\left(\sum_{|n|=1}^{\infty} k(x, n) b_{n}\right) f(x) d x,
$$

then we can rewrite (33) and (35) as follows:

$$
\begin{aligned}
\left(T^{(2)} b, f\right) & <\left\|T^{(2)}\right\| \cdot\|f\|_{p, \Psi}\|b\|_{q, \Phi}, \\
\left\|T^{(2)} b\right\|_{q, \Phi^{1-q}} & <\left\|T^{(2)}\right\| \cdot\|b\|_{q, \Psi} .
\end{aligned}
$$

Remark 9. (i) For $\delta=-1$, (36) reduces to (5). If $f(-x)=$ $f(x)(x>0)$ and $b_{-n}=b_{n}(n \in \mathbf{N})$, then (5) reduces to the following half-discrete Hilbert-type inequality:

$$
\begin{aligned}
\sum_{n=1}^{\infty} \int_{0}^{\infty} \frac{1}{1+\rho(n x)^{\gamma}} f(x) b_{n} d x \\
\quad<\frac{\pi}{\gamma \rho^{\sigma / \gamma} \sin \pi(\sigma / \gamma)}\left[\int_{0}^{\infty} x^{p(1-\sigma)-1} f^{p}(x) d x\right]^{1 / p} \\
\quad \cdot\left[\sum_{n=1}^{\infty} n^{q(1-\sigma)-1} b_{n}^{q}\right]^{1 / q} .
\end{aligned}
$$

(ii) For $\delta=1,(33)$ reduces to the following particular inequality with the homogeneous kernel of degree 0 :

$$
\begin{aligned}
& \sum_{|n|=1}^{\infty} \int_{-\infty}^{\infty} \frac{f(x) b_{n}}{1+\rho[(|n|+n \cos \beta) /(|x|+x \cos \alpha)]^{\gamma}} d x \\
& <\frac{2 \pi \csc ^{2 / q} \alpha \csc ^{2 / p} \beta}{\gamma \rho^{\sigma / \gamma} \sin \pi(\sigma / \gamma)}\left\{\int_{-\infty}^{\infty}(|x|+x \cos \alpha)^{p(1+\sigma)-1}\right. \\
& \left.\cdot f^{p}(x) d x\right\}^{1 / p} \cdot\left\{\sum_{|n|=1}^{\infty}(|n|+n \cos \beta)^{q(1-\sigma)-1}\right. \\
& \left.\cdot b_{n}^{q}\right\}^{1 / q} .
\end{aligned}
$$

(iii) For $\delta=-1$, (33) reduces to the following particular inequality with the nonhomogeneous kernel:

$$
\begin{aligned}
& \sum_{|n|=1}^{\infty} \int_{-\infty}^{\infty} \frac{f(x) b_{n}}{1+\rho[(|x|+x \cos \alpha)(|n|+n \cos \beta)]^{\gamma}} d x \\
& <\frac{2 \pi \csc ^{2 / q} \alpha \csc ^{2 / p} \beta}{\gamma \rho^{\sigma / \gamma} \sin \pi(\sigma / \gamma)}\left\{\int_{-\infty}^{\infty}(|x|+x \cos \alpha)^{p(1-\sigma)-1}\right. \\
& \left.\cdot f^{p}(x) d x\right\}^{1 / p} \cdot\left\{\sum_{|n|=1}^{\infty}(|n|+n \cos \beta)^{q(1-\sigma)-1}\right. \\
& \left.\cdot b_{n}^{q}\right\}^{1 / q} .
\end{aligned}
$$

The constant factors in (5) and the above inequalities are all the best possible ones.

\section{Competing Interests}

The authors declare that they have no competing interests.

\section{Authors' Contributions}

Bicheng Yang carried out the mathematical studies, participated in the sequence alignment, and drafted the manuscript. Qunwei Ma and Leping He participated in the design of the study and performed the numerical analysis. All authors read and approved the final manuscript. 


\section{Acknowledgments}

This work is supported by Jishou University Graduate Student Research Innovation Project in 2016 (no. JGY201644) and Hunan Province Natural Science Foundation (no. 2015JJ4041). The authors are grateful for their help.

\section{References}

[1] G. H. Hardy, "Note on a theorem of Hilbert concerning series of positive terms," Proceedings of the London Mathematical Society, vol. 23, no. 2, 1925.

[2] G. H. Hardy, J. E. Littlewood, and G. Pólya, Inequalities, Cambridge University Press, Cambridge, Mass, USA, 1934.

[3] B. C. Yang, "A half-discrete Hilbert's inequality," Journal of Guangdong University of Education, vol. 31, no. 3, pp. 1-7, 2011.

[4] D. S. Mitrinović, J. E. Pecaric, and A. M. Fink, Inequalities Involving Functions and Their Integrals and Deivatives, Kluwer Academic, Boston, Mass, USA, 1991.

[5] B. C. Yang and L. Debnath, Half-Discrete Hilbert-Type Inequalities, World Scientific, Singapore, 2014.

[6] B. C. Yang, "A survey of the study of Hilbert-type inequalities with parameters," Advances in Mathematics, vol. 38, no. 3, pp. 257-268, 2009.

[7] J. S. Xu, "Hardy-Hilbert's inequalities with two parameters," Advances in Mathematics, vol. 36, no. 2, pp. 63-76, 2007.

[8] D. M. Xin, "A Hilbert-type integral inequality with the homogeneous kernel of zero degree," Mathematical Theory and Applications, vol. 30, no. 2, pp. 70-74, 2010.

[9] M. Krnic and J. E. Pecaric, "Hilbert's inequalities and their reverses," Publicationes Mathematicae Debrecen, vol. 67, no. 34, pp. 315-331, 2005.

[10] Y. Hong, "On Hardy-Hilbert integral inequalities with some parameters," Journal of Inequalities in Pure and Applied Mathematics, vol. 6, no. 4, article 92, 10 pages, 2005.

[11] I. Brnetić and J. Pečarić, "Generalization of Hilbert's integral inequality," Mathematical Inequalities \& Applications, vol. 7, no. 2, pp. 199-205, 2004.

[12] M. Krnic and J. E. Pecaric, "General Hilbert's and Hardy's inequalities," Mathematical Inequalities \& Applications, vol. 8, no. 1, pp. 29-51, 2005.

[13] B. Arpad and O. Choonghong, "Best constants for certain multilinear integral operators," Journal of Inequalities and Applications, vol. 2006, Article ID 28582, 12 pages, 2006.

[14] J. Kuang and L. Debnath, "On Hilbert's type inequalities on the weighted Orlicz spaces," Pacific Journal of Applied Mathematics, vol. 1, no. 1, pp. 95-103, 2007.

[15] Y. Li and B. He, "On inequalities of Hilbert's type," Bulletin of the Australian Mathematical Society, vol. 76, no. 1, pp. 1-13, 2007.

[16] Q. Huang, "A new extension of a Hardy-Hilbert-type inequality," Journal of Inequalities and Applications, vol. 2015, no. 1, article 397, 2015.

[17] B. He and Q. Wang, "A multiple Hilbert-type discrete inequality with a new kernel and best possible constant factor," Journal of Mathematical Analysis and Applications, vol. 431, no. 2, pp. 889902, 2015.

[18] G. V. Milovanović and M. T. Rassias, "Some properties of a hypergeometric function which appear in an approximation problem," Journal of Global Optimization, vol. 57, no. 4, pp.11731192, 2013.
[19] G. V. Milovanovic and M. T. Rassias, Eds., Analytic Number Theory, Approximation Theory, and Special Functions, Springer, New York, NY, USA, 2014.

[20] B. C. Yang, "A new Hilbert-type integral inequality," Soochow Journal of Mathematics, vol. 33, no. 4, pp. 849-859, 2007.

[21] Z. Zeng and Z. T. Xie, "On a new hilbert-type intergral inequality with the intergral in whole plane," Journal of Inequalities and Applications, vol. 2010, Article ID 256796, 8 pages, 2010.

[22] Z. Zeng, K. Raja Rama Gandhi, and Z. T. Xie, "A new Hilberttype inequality with the homogeneous kernel of degree -2 and with the integral," Bulletin of Mathematical Sciences of Applications, vol. 3, no. 1, pp. 11-20, 2014.

[23] B. C. Yang and Q. Chen, "On a Hardy-Hilbert-type inequality with parameters," Journal of Inequalities and Applications, vol. 2015, article 339, 2015.

[24] J. C. Kuang, Applied Inequalities, Shangdong Science and Technology Press, Jinan, China, 2004.

[25] J. C. Kuang, Real and Functional Analysis, Higher Education Press, Beijing, China, 2nd edition, 2015. 


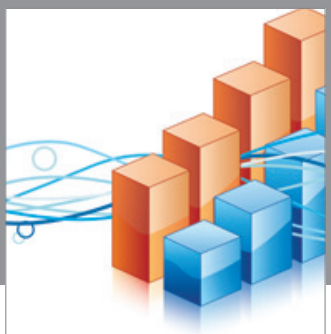

Advances in

Operations Research

vatem alat4

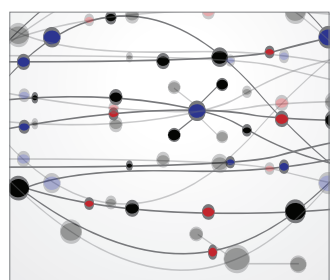

\section{The Scientific} World Journal
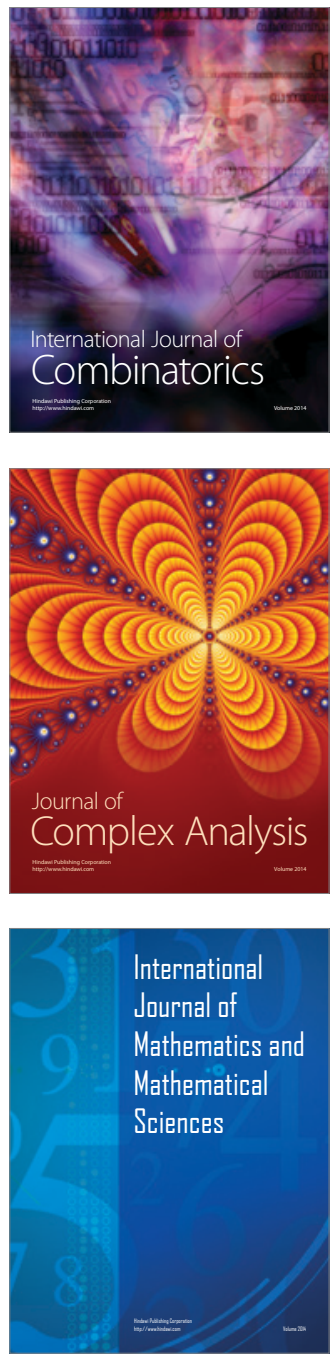
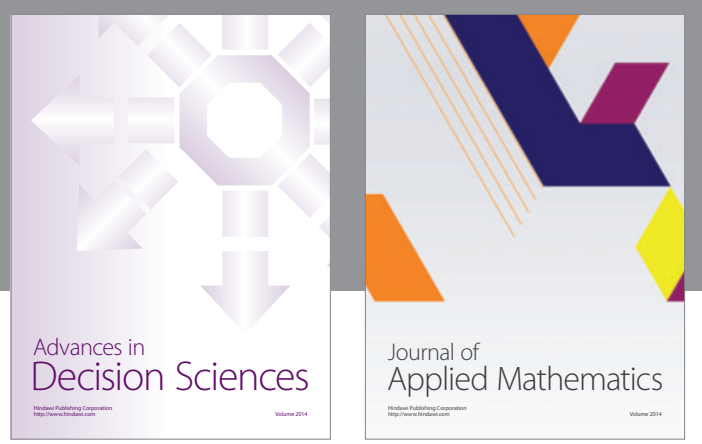

Algebra

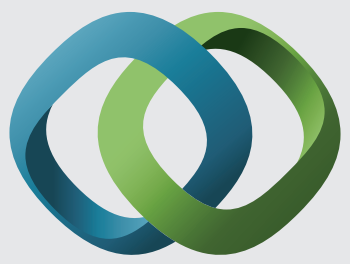

\section{Hindawi}

Submit your manuscripts at

http://www.hindawi.com
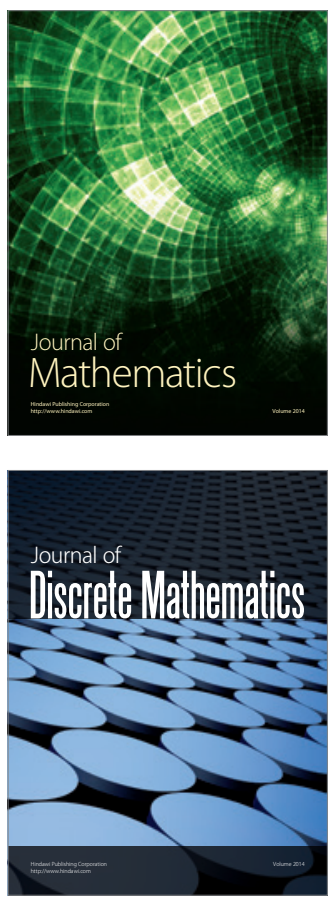

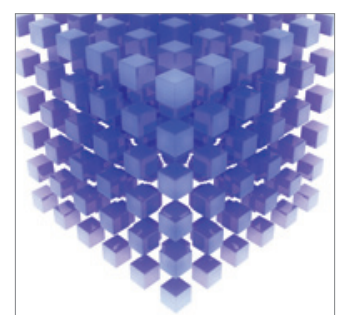

Mathematical Problems in Engineering
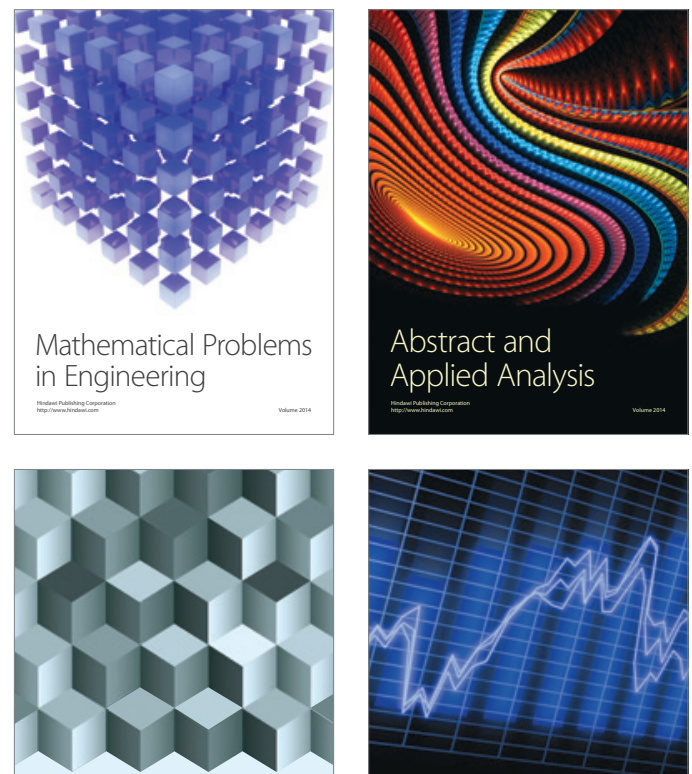

Journal of

Function Spaces

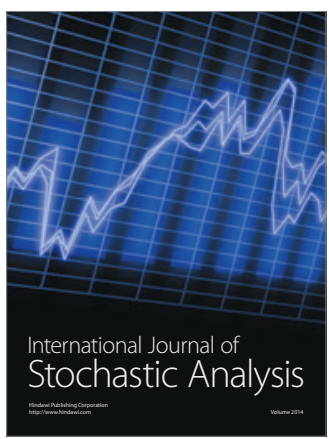

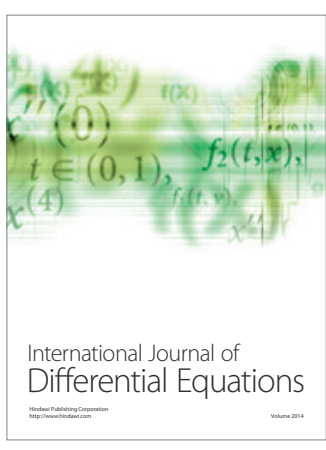
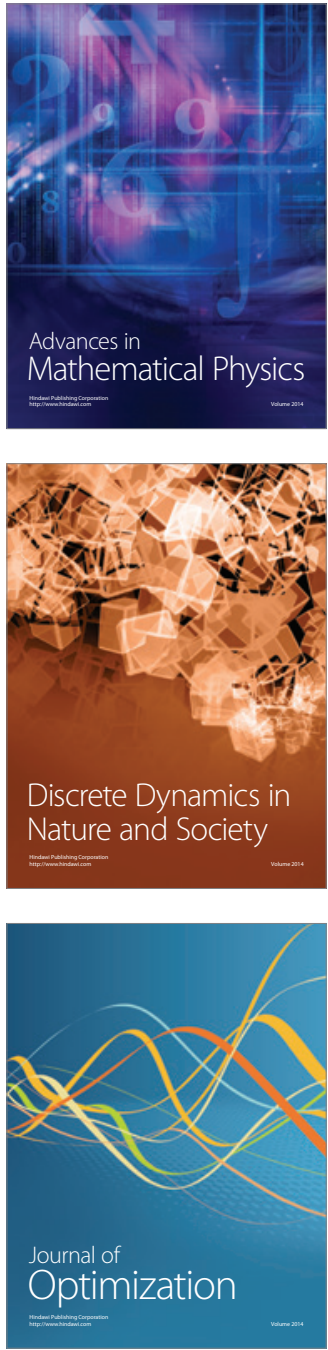